\title{
Standardization of Agrotechniques and Biochemical Assessment of Crataegus oxyacantha in Western Himalaya
}

\author{
Gopichand $^{1 *}$, RL Meena' ${ }^{1}$ P Kaur ${ }^{2}$, RD Singh ${ }^{1}$
}

Gopichand ${ }^{1 *}$, RL Meena' ${ }^{1}$, P Kaur $^{2}$, RD Singh ${ }^{1}$

'Department of High Altitude Biology, CSIR - Institute of Himalayan Bioresource Technology, Palampur (H.P.) 176061 INDIA.

${ }^{2}$ Sri Guru Gobind Singh College, Sector-26, Chandigarh-160019, INDIA.

Correspondence

Gopichand

CSIR - Institute of Himalayan Bioresource Technology, Palampur (H.P.) 176061 INDIA.

Phone no: +91-1894-233339

E-mail: gopichand@ihbt.res.in

History

- Submission Date: 17-08-2017;

- Review completed: 11-09-2017;

- Accepted Date: 02-11-2017

DOI : 10.5530/pj.2017.6s.160

Article Available online http://www.phcogj.com/v9/i6s

\section{Copyright}

(c) 2017 Phcog.Net. This is an openaccess article distributed under the terms of the Creative Commons Attribution 4.0 International license.

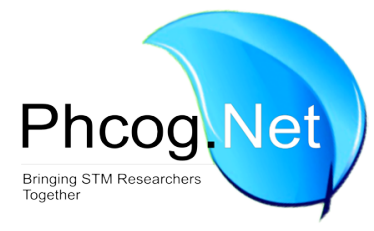

\begin{abstract}
C. oxyacantha is a high valued medicinal plant of Rosacea family. It is used to cure cardiac disorder in ayurvedic medicines. A field experiment was laid out in 2004 in CSIR-IHBT farm, by using different quantity of FYM and various spacing. Low growth in plant height was observed in first five years with higher dose of FYM, but in 2015 the significant height growth was recorded. From 2008 to 2015 all types of FYM applications produced statistically significant yield of seed production except in 2012 and 2014. The 22.50t/ha was the most statistically significant dose of FYM in relation to seed yield. The spacing did not produce any significant results for seed production. A vegetative propagation trial of $C$. oxyacantha was also laid out using semi hard stem cuttings and some selected hormones (IAA, IBA, GA and Abscisic acid) with different concentrations. Statistically significant shoot sprouting (78.35\%) was recorded when IBA of $1000 \mathrm{mg} / \mathrm{L}$ was used followed by $67.74 \%$ in case of $1500 \mathrm{mg} / \mathrm{L}$ of the same hormone. While lowest shoot sprouting $(27.85 \%)$ was observed using $2000 \mathrm{mg} / \mathrm{L}$ of Abscisic acid. A statistically significant $5.67 \mathrm{~cm}$ and $5.33 \mathrm{~cm}$ shoot lengths were observed using $2000 \mathrm{mg} / \mathrm{L}$ of IAA and $1000 \mathrm{mg} / \mathrm{L}$ of IBA, respectively. In the case of shoot tillers 3.33 was recorded in $1500 \mathrm{mg} / \mathrm{l}$. of IAA. Two new compounds and 9 known compounds were isolated from fruit extract.
\end{abstract}

Key words: Crataegus oxyacantha, Hormones, Bioactive compounds, FYM, Spacing.

\section{INTRODUCTION}

Crataegus oxyacantha (hawthorn) belongs to the family Rosacea. It is a middle deciduous spiny tree, distributed in Himalaya from 2500 to $4000 \mathrm{~m}$ amsl or near the tree lines of western Himalaya. In India, it is an endangered species. It is a native of Europe and North America. It's all parts such as bark, flower and fruits have medicinal importance, especially in cardiac disorders and leaves are used in herbal tea. Its cardiac and arrhythmia curing properties were studied. ${ }^{1}$ Some workers have also reported its role in controlling various diseases like myocardial problem, ${ }^{2}$ heart failure, ${ }^{3}$ antioxidant, anti-inflammatory and antimicrobial infections. ${ }^{4,5}$ It is also used in some other critical disease such as control of central nervous system (CNS), anxiety and depression. ${ }^{6}$ A Homeopathic medicine has also been prepared from its flowers and fruits by Homeopathic pharmacopeia of India. ${ }^{7}$ As food for heart has described. ${ }^{8}$ Some of its phenolic constituents like hyperoxide, vitexin, quercetin and rutin, epicatechin, catechin and phenolic acids have pharmacological properties. $^{\text {? }}$

\section{MATERIAL AND METHODS}

Field experiments were conducted from 2004 to 2016 in High Altitude Biology division farm of the CSIR-Institute of Himalayan Bioresource Technology
(IHBT), Palampur (1325 m. amsl. 32006’0”N, $76^{\circ}$ $\left.34^{\prime} 10^{\prime \prime} \mathrm{E}\right)$. The weather data was recorded by CSKHPKV Palampur Table 1. The soil of the experimental field is silty clay loam in texture acidic in reaction ( $\mathrm{pH}$ 6.4) high in organic carbon (2.4\%) low level of $\mathrm{N}$ (198ha-1) medium in available $\mathrm{P}(23 \mathrm{~kg})$ and available K (538 kgha-1).

In agro-climatic conditions of Palampur, C. oxyacantha trees defoliated from the month of October and new shoot emerged in $1^{\text {st }}$ week of April. Flowering also started within a week. Seed formation started immediately after 15-20 days of flowering. The seed matured in the month of July. When seed became red in color, its plucking was started. All the seed were plucked up to the end of August month. No disease has been reported in this plantation so far from July 2004. The seed formation though started in 2006 , but it was properly started in 2007. Since then various growth parameters were recorded and presented in various tables. Statistically analyses have also been carried out every year.

\section{Agrotechniques \\ Field experiment}

An experiment was laid out for standardization the agrotechniques of C. oxyacantha. The stem cuttings 
were used for vegetative propagation. The diameters of stem cuttings were 6-8 $\mathrm{mm}$ and $8-10 \mathrm{~cm}$ which were less then pencil thickness.

The lower portions of stem cuttings ( 2 inch) were dipped in different solutions up to $3 \mathrm{~h}$ and the control was dipped in plane water. In the experiment some selected hormones, IAA, IBA, GA 3 and Abscisic acid was used with the concentrations of 1000,1500 and $2000 \mathrm{mg} / \mathrm{l}, 100 \mathrm{no}$. stem cuttings were used for each concentration, it was replicate thrice time i.e. 300 no. stem cuttings for each concentration in polytunnel. The sprouting time was taken about 60 to 80 days. The mature seed was collected in September 2015; the seeds were dried in natural open field for three months and at $-5^{\circ} \mathrm{C}$ in cold chamber. After 3 months, the seed were pickup outside the cold chamber. In the case of seed, the dormancy period is two years so, it is very difficult and time consuming. So, the vegetative propagation is preferred by shoot cuttings for raising sapling of C. oxyacantha. In two-year time most of the seeds were damaged or infected by some and other causes in the soil and in a store.

\section{Nursery techniques}

The land was prepared well by manual digging, levelling, breaking soil clods, weeding and finally mixing it with well rotten FYM and sands. The semi hard brown stem cuttings were collected from our own field. These cuttings were $8-10 \mathrm{~cm}$ long and 6-8 $\mathrm{mm}$ in diameter with 5-6 nodes. The stem cuttings were collected in the last week of January 2016. The experiment laid out on 02.02.2016. The stem cutting was dipped in solution of selected hormones IAA, IBA, GA 3 and Abscisic acid for $3 \mathrm{~h}$. The stem cuttings planted at $10.0 \mathrm{~cm} \times 10.0 \mathrm{~cm}$ spacing. All the used hormones promoting callus. Normally stem cuttings take 70-80 days for rooting. The rooted cuttings were hardened for 2 months before transplanting in the field. The water was given as or when required. The reading of sprouting of sprouts stem cuttings were recorded in last week of March and $1^{\text {st }}$ week of April 2016.

\section{Soil type}

C. oxyacantha is a middle tree and deciduous in nature. It is very well grown in sandy, clay-loam soil and sub humid, temperate climate. It's like low or medium clay-loam soil.

\section{Land preparation and manure application}

First of all, wild bushes with rooted stock and secondary roots were removed. Tractor tillage 4-5 times with disc than cultivator and the soil was leveled by leveler. The soil texture should be fine without any clods. FYM 15t/ha was thoroughly mixed, some quantity of sand was also mixed in soil to make it porous in nature. For plantation 45.0 x 45.0 $\mathrm{x} 45.0 \mathrm{~cm}$ dimension pits were dugout. In the experiment, FYM application $15 \mathrm{t} / \mathrm{ha}, 22.5 \mathrm{t} / \mathrm{ha}, 30 \mathrm{t} / \mathrm{ha}$ and $37.5 \mathrm{t} / \mathrm{ha}$ was applied, each plot was replicated 4 times. The FYM applications were given before planting and were repeated every year in the month of December. Because it is a deciduous middle tree, there was no any growth observed during October to March. The growth period was April to October. In the month of December FYM was very well mixed by manually by proper digging with phawra. In dormant period, the loss of roots very less and recovered subsequently. The five spacing S1-1.0x1.0 m, S2-2.0x1.0m, S3-2.0x2.0 m, S4-3.0x1.0 m and S5-3.0x2.0 m was applied for this experiment with 3 no. replications. The planting has been done in the month of July 2004. And some filling was covered in August 2004. Because it is hilly moist region, wild bushes and unwanted vegetation including weeds were frequently come up.

In all the treatments, plants were planted at the given spacing. At the time of planting, the soil sample of top soil and sub soil (15 cm depth) were taken and analyzed for physic-chemical properties. The soil was silty clay loam in texture. Normally acidic in nature, high organic carbon percentage, available NPK high and average.

\section{Isolation of bioactive compounds from Crataegus oxyacantha fruit extract. Collection of plant material}

The fruits of Crataegus oxyacantha were collected from IHBT Institute's farm in the month of July. The fruits were shade dried for 15 days and then in hot air oven at $30-40^{\circ} \mathrm{C}$. The fruits were pulverized electronic grinder machine in to coarse powder. The voucher specimens (PLP 007) collected was identified, processed and deposited in the Herbarium of CSIR-IHBT, Palampur, India.

\section{Extraction and fractionation of plant material}

The powdered material of fruits $(13 \mathrm{~kg})$ was percolated four times with $70 \%$ aqueous ethanol at room temperature and the extract was concentrated for further studies on rotary evaporator at $40^{\circ} \mathrm{C}$. The fruit extract was found to be $1391.2 \mathrm{~g}$. For fractionation, crude extract (600 $\mathrm{g}$ for fruit) was defatted with $n$-hexane and remaining extract was partitioned with ethyl acetate and $n$-butanol. The respective ethyl acetate and $n$-butanol extracts obtained were 128.4 (FE) and $180.9 \mathrm{~g}$ (FB).

\section{General experimental procedure}

Melting points were determined on a Bronsted Electrothermal 9100. Mass spectra were recorded on QTOF-Micro of Waters Micro mass. NMR experiments were performed on Bruker Avance-300 spectrometer. For open column chromatography (OCC) silica gel (60-120 mesh), for dry column chromatography (DCC) silica gel-H and for flash column chromatography (FCC) silica gel (230-400 mesh) was used. Thin layer chromatography (TLC) was performed on silica gel G (on glass plates) and precoated silica gel $60 \mathrm{~F}_{254}$ plates. The detection of chemical constituent's present in the extract/fractions was carried out by iodine vapours, UV-detection and spraying reagents. The solvent systems used to resolve the chemical constituents and spraying reagents used for the detection are summarized below:

\section{Isolation of secondary metabolites}

The ethyl acetate fraction of fruit extract (FE) was subjected to DCC using $\mathrm{CH}_{2} \mathrm{Cl}_{2}: \mathrm{MeOH}: \mathrm{H}_{2} \mathrm{O}$ (100:13.5:16.5) as eluting solvent and seven fractions (Fr.) from FEa-FEg were collected on the basis of TLC pattern. The Fr. FEa was re-chromatographed using OCC with $\mathrm{CH}_{2} \mathrm{Cl}_{2}: \mathrm{MeOH}$ : $\mathrm{H}_{2} \mathrm{O}(100: 13.5: 16.5)$ and again seven Fr. (FEa1-FEa7) were obtained. FEa1 yielded colourless needles of $\beta$-sitosterol 1 (40 mg), whereas, $\beta$-sitosterol-3-O- $\beta$-D-glucoside $2(31 \mathrm{mg})$ was obtained from FEa6. Similarly, $\mathrm{FEb}$ was subjected to OCC in $\mathrm{CH}_{2} \mathrm{Cl}_{2}$ with increasing polarity of $\mathrm{MeOH}$ and 35 fractions of $25 \mathrm{ml}$ each were collected. Fr. 4-10 yielded oleanolic acid $3(15 \mathrm{mg})$ whereas ursolic acid $4(67 \mathrm{mg})$ was purified from Fr. 19-32 using reverse phase silica $\left(\mathrm{MeOH}-\mathrm{H}_{2} \mathrm{O}\right)$. A white precipitate of bis-(2-ethylhexyl) phthalate 5 ( $19 \mathrm{mg}$ ) was obtained from FEc. The Fr. FEe was re-chromatographed using FCC in isocratic mode with $\mathrm{CH}_{2} \mathrm{Cl}_{2}: \mathrm{MeOH}: \mathrm{H}_{2} \mathrm{O}$ (100:18:20) and five fractions (FEe1-FEe5) based on TLC were obtained. FEe1 was re-chromatographed over silica using OCC with $\mathrm{CH}_{2} \mathrm{Cl}_{2}: \mathrm{MeOH}: \mathrm{H}_{2} \mathrm{O}$ (100:13.5:16.5) to yield quercetin 6 (10 mg) and $(2 R, 3 R)$-taxifolin $7(14 \mathrm{mg})$ as yellow and brownish yellow powder, respectively.

The $n$-butanol fraction (FB) of fruit extract was subjected to DCC using EtOAc: $\mathrm{CHCl}_{3}: \mathrm{MeOH}: \mathrm{H}_{2} \mathrm{O}(15: 8: 4: 1)$ in isocratic manner and nine fractions (FBa-FBi) were collected on the basis of TLC. Fr. FBc was re-chromatographed over FCC using EtOAc: $\mathrm{CHCl}_{3}: \mathrm{MeOH}: \mathrm{H}_{2} \mathrm{O}$ (15:8:1:0.5 to $15: 8: 2: 0.5)$ and five fractions were obtained. The Fr. 3 yielded vitexin-2"-O-rhamnoside $8(32 \mathrm{mg})$ whereas, Fr. 5 afforded 
Table 1: Weather parameters during 2004 to 2016 recorded at CSKHPKV, Palampur (H.P.)

\begin{tabular}{|c|c|c|c|c|c|c|}
\hline \multirow[t]{2}{*}{ Years } & \multirow{2}{*}{$\begin{array}{c}\text { Temperature } \\
\text { Maximum }\left({ }^{\circ} \mathrm{C}\right)\end{array}$} & \multirow{2}{*}{$\begin{array}{c}\text { Temperature } \\
\text { Minimum }\left({ }^{\circ} \mathrm{C}\right)\end{array}$} & \multicolumn{2}{|c|}{ Relative Humidity (\%) } & \multirow{2}{*}{$\frac{\text { Bright Sun }}{\text { shine hrs }}$} & \multirow{2}{*}{$\frac{\text { Rainfall }}{\mathrm{mm}}$} \\
\hline & & & RH (Max) & RH (Min) & & \\
\hline 2004 & 24.90 & 13.40 & 65.20 & 55.00 & 384.50 & 2206.40 \\
\hline 2005 & 20.08 & 10.90 & 59.23 & 54.15 & 345.80 & 2860.80 \\
\hline 2006 & 20.18 & 11.83 & 55.12 & 46.25 & 343.80 & 2730.20 \\
\hline 2007 & 19.86 & 11.19 & 57.61 & 45.54 & 359.30 & 1848.60 \\
\hline 2008 & 19.65 & 10.88 & 56.90 & 47.20 & 328.40 & 2304.80 \\
\hline 2009 & 20.47 & 10.61 & 59.49 & 45.84 & 348.00 & 1768.40 \\
\hline 2010 & 25.62 & 13.52 & 73.57 & 56.23 & 345.44 & 2569.60 \\
\hline 2011 & 23.90 & 13.17 & 81.31 & 67.24 & 331.41 & 2500.60 \\
\hline 2012 & 24.01 & 12.81 & 70.96 & 56.18 & 348.23 & 2421.99 \\
\hline 2013 & 23.55 & 13.05 & 75.55 & 61.18 & 328.41 & 3142.00 \\
\hline 2014 & 23.30 & 12.41 & 72.74 & 59.15 & 327.62 & 1886.10 \\
\hline 2015 & 23.65 & 12.53 & 77.72 & 64.41 & 318.30 & 2630.80 \\
\hline 2016 & 24.61 & 12.55 & 75.26 & 60.22 & 345.19 & 2230.80 \\
\hline
\end{tabular}

Table 2: Effect of growth hormones on shoot sprouting of Crataegus oxyacantha.

\begin{tabular}{|c|c|c|c|c|}
\hline PGR mg/l & Sprout Cutting no. & Sprouting \% & Tiller No. & Plant Height $\mathrm{cm}$ \\
\hline IAA-1000 & 40.00 & 47.62 & 2.67 & 2.67 \\
\hline IAA-1500 & 35.33 & 56.08 & 3.33 & 4.00 \\
\hline IAA-2000 & 39.00 & 51.53 & 2.67 & 5.67 \\
\hline IBA-1000 & 60.33 & 78.35 & 1.67 & 5.33 \\
\hline IBA-1500 & 42.00 & 67.74 & 2.00 & 3.33 \\
\hline IBA-2000 & 46.67 & 52.03 & 2.00 & 3.33 \\
\hline $\mathrm{GA}_{3}-1000$ & 37.33 & 50.45 & 2.33 & 5.00 \\
\hline $\mathrm{GA}_{3}-1500$ & 39.00 & 55.71 & 2.00 & 4.00 \\
\hline $\mathrm{GA}_{3}-2000$ & 45.00 & 53.57 & 3.00 & 3.33 \\
\hline Abscsic Acid-2000 & 26.67 & 27.85 & 1.67 & 2.67 \\
\hline SEm \pm & 2.85 & 3.72 & 0.42 & 0.50 \\
\hline $\mathrm{CD}$ at $5 \%$ & 8.48 & 11.05 & NS & 1.48 \\
\hline
\end{tabular}

(2R,3R)-taxifolin-3-O- $\beta$-D-xylopyranoside 9 (16 mg). The fraction Fbe was chromatographed over reverse phase silica $\left(\mathrm{MeOH}-\mathrm{H}_{2} \mathrm{O}\right)$ which provided isoquercitrin $10(21 \mathrm{mg})$ and it was purified with sephadexLH-20. The yellow precipitates of rutin $11(11 \mathrm{mg})$ were obtained from FBf.

\section{RESULTS}

The results of seed germination were poor (3.7\%) and statistically nonsignificant on higher application of hormones. In case of semi hard stem cutting, which were used for vegetative propagation by using some selected hormone treatments, the sprouting time was 60-80 days Figure 1A. The statistically significant results $(78.35 \%)$ was recorded in case of $1000 \mathrm{mg} / \mathrm{l} \mathrm{IBA}$ followed by $67.74 \%$ growth using $1500 \mathrm{mg} / \mathrm{l} \mathrm{IBA}$. In the case of shoot tiller parameters, it was maximum in $1500 \mathrm{mg} / \mathrm{l} \mathrm{IAA}$ and minimum in Abscisic acid 1.67 number. But these results were statistically non-significant. In terms of plant height IAA produced statistically significant $5.67 \mathrm{~cm}$, and parallel in IBA $1000 \mathrm{mg} / \mathrm{l}$, followed by $\mathrm{GA}_{3}$ $100 \mathrm{mg} / \mathrm{l}$ Table 2.

The plant height was recorded during 2005 to 2009 and lastly in 2015 . All the results were statistically analyzed and found non-significant Table 3. The FYM application could not influence the plant height in all the years.
However, the seed were produced in 2006, but the yield was very low. In the year 2007 also, the seed yield was recorded statistically nonsignificant. But from 2008 to 2011, it was statistically significant Table 4 . Again in 2012 and 2014, the seed yield was non-significant. In 2015, all FYM applications produced higher and statistically significant yield as compared to other years. In 2016 the statistically significant seed yield was recorded. It was observed that the seed yield was highly affected by local weather conditions. At the time of flowering and seed setting, the high wind, high rainfall and big size hailstorms highly affected the seed yield leading to statistically non-significant result. In all observations, the plant spacing did not affect the plant growth as well as seed yield Table $6,7,8$, but the dose of FYM produced high and statistically significant results Table 4,5. For commercial utilization, these harvested seeds were dried under shade conditions Figure 1B, C,D,E. To avoid moisture, the seed were dried in aluminum trays (iron mesh) and every alternate day the seed were transferred and changed to its previous position. The dried seeds are weighed and stored in jute bags, which are now ready for sale. Two new compounds from Crataegus sp. and 9 known compounds were isolated from the ethyl acetate and n-butanol fractions of fruit extract of Crataegus oxyacantha. The new compounds are Bis-(2-ethylhexyl) phthalate and $(2 R, 3 R)$-Taxifolin-3-O- $\beta$-D-xylopyranoside; whereas, the 
Table 3: Crataegus oxyacantha FYM trial observation for plant height.

\begin{tabular}{ccccccc}
\hline FYM & $\begin{array}{c}\text { Plant height } \\
(\mathbf{c m}) \mathbf{2 0 0 5}\end{array}$ & $\begin{array}{c}\text { Plant height }(\mathbf{c m}) \\
\text { 2006 }\end{array}$ & $\begin{array}{c}\text { Plant height }(\mathbf{c m}) \\
\text { 2007 }\end{array}$ & $\begin{array}{c}\text { Plant height }(\mathbf{c m}) \\
\text { 2008 }\end{array}$ & $\begin{array}{c}\text { Plant height }(\mathbf{c m}) \\
\text { 2009 }\end{array}$ & $\begin{array}{c}\text { Plant height }(\mathbf{c m}) \\
\mathbf{2 0 1 5}\end{array}$ \\
\hline $\mathbf{1 5 t} / \mathbf{h a}$ & 173.58 & 225.00 & 230.99 & 297.92 & 346.69 & 450.00 \\
$\mathbf{2 2 . 5 t} / \mathbf{h a}$ & 159.67 & 210.45 & 242.54 & 291.25 & 357.19 & 425.42 \\
$\mathbf{3 0 t / h a}$ & 175.71 & 227.79 & 238.06 & 268.33 & 330.94 & 407.50 \\
$\mathbf{3 7 . 5 t} / \mathbf{h a}$ & 156.79 & 218.21 & 230.19 & 283.00 & 322.19 & 425.83 \\
SEm \pm & 5.07 & 7.09 & 8.95 & 11.74 & 24.61 & 9.19 \\
CD at 5\% & NS & NS & NS & NS & NS & NS \\
\hline
\end{tabular}

Table 4: Crataegus oxyacantha FYM trial observation for seed weight (kg/plot).

\begin{tabular}{cccccc}
\hline FYM & $\begin{array}{c}\text { Seed weight } \\
(\mathbf{k g}) \mathbf{2 0 0 7}\end{array}$ & $\begin{array}{c}\text { Seed weight } \\
(\mathbf{k g}) \mathbf{2 0 0 8}\end{array}$ & $\begin{array}{c}\text { Seed weight } \\
(\mathbf{k g}) \mathbf{2 0 0 9}\end{array}$ & $\begin{array}{c}\text { Seed weight } \\
(\mathbf{k g}) \mathbf{2 0 1 0}\end{array}$ & $\begin{array}{c}\text { Seed weight } \\
(\mathbf{k g}) \mathbf{2 0 1 1}\end{array}$ \\
\hline $\mathbf{1 5 t / h a}$ & 0.45 & 0.98 & 0.41 & 4.66 & 12.20 \\
$\mathbf{2 2 . 5 t / h a}$ & 0.97 & 1.10 & 0.48 & 4.80 & 16.69 \\
$\mathbf{3 0 t / h a}$ & 0.67 & 0.84 & 0.43 & 6.18 & 16.89 \\
$\mathbf{3 7 . 5 t / h a}$ & 0.51 & 1.42 & 0.34 & 7.81 & 14.36 \\
$\mathbf{S E m} \pm$ & 0.30 & 0.24 & 0.06 & 0.84 & 1.58 \\
$\mathbf{C D}$ at 5\% & NS & 0.77 & 0.19 & 2.69 & 5.07 \\
\hline
\end{tabular}

Table 5: Crataegus oxyacantha FYM trial observation total seed weight kg/plot.

\begin{tabular}{cccccc}
\hline FYM & $\begin{array}{c}\text { Seed weight } \\
(\mathbf{k g}) \mathbf{2 0 1 2}\end{array}$ & $\begin{array}{c}\text { Seed weight } \\
(\mathbf{k g}) \mathbf{2 0 1 3}\end{array}$ & $\begin{array}{c}\text { Seed weight } \\
(\mathbf{k g}) \mathbf{2 0 1 4}\end{array}$ & $\begin{array}{c}\text { Seed weight } \\
(\mathbf{k g}) \mathbf{2 0 1 5}\end{array}$ & $\begin{array}{c}\text { Seed weight } \\
(\mathbf{k g}) \mathbf{2 0 1 6}\end{array}$ \\
\hline $\mathbf{1 5 t} / \mathbf{h a}$ & 18.08 & 12.44 & 3.13 & 46.00 & 8.00 \\
$\mathbf{2 2 . 5 t / h a}$ & 24.35 & 17.69 & 4.44 & 54.50 & 8.13 \\
$\mathbf{3 0 t / h a}$ & 27.00 & 20.31 & 4.81 & 48.00 & 7.75 \\
$\mathbf{3 7 . 5 t} / \mathbf{h a}$ & 22.13 & 11.75 & 3.19 & 48.25 & 5.50 \\
$\mathbf{S E m} \pm$ & 3.25 & 2.53 & 0.77 & 4.35 & 1.75 \\
$\mathbf{C D}$ at $\mathbf{5 \%}$ & NS & 8.09 & NS & 13.93 & 5.60 \\
\hline
\end{tabular}

Table 6: Crataegus oxyacantha spacing trial observation for plant height.

\begin{tabular}{|c|c|c|c|c|c|c|}
\hline Spacing & $\begin{array}{c}\text { Plant height }(\mathrm{cm}) \\
2005\end{array}$ & $\begin{array}{l}\text { Plant height }(\mathrm{cm}) \\
2006\end{array}$ & $\begin{array}{l}\text { Plant height }(\mathrm{cm}) \\
2007\end{array}$ & $\begin{array}{c}\text { Plant height }(\mathrm{cm}) \\
2008\end{array}$ & $\begin{array}{c}\text { Plant height }(\mathrm{cm}) \\
2009\end{array}$ & $\begin{array}{l}\text { Plant height }(\mathrm{cm}) \\
2015\end{array}$ \\
\hline $\mathrm{S} 1=1 \times 1 \mathrm{~m}$ & 169.99 & 207.90 & 229.42 & 275.00 & 359.56 & 444.86 \\
\hline $\mathrm{S} 2=2 \times 1 \mathrm{~m}$ & 172.25 & 205.78 & 223.00 & 299.03 & 335.42 & 453.19 \\
\hline $\mathrm{S} 3=2 \times 2 \mathrm{~m}$ & 176.56 & 212.89 & 229.55 & 280.00 & 324.58 & 424.58 \\
\hline $\mathrm{S} 4=3 \times 1 \mathrm{~m}$ & 181.38 & 212.42 & 234.72 & 297.29 & 336.83 & 425.14 \\
\hline $\mathrm{S} 5=3 \times 2 \mathrm{~m}$ & 176.17 & 223.33 & 236.00 & 262.50 & 278.33 & 469.31 \\
\hline SEm \pm & 9.04 & 11.51 & 13.13 & 10.90 & 17.88 & 18.14 \\
\hline CD at $5 \%$ & NS & NS & NS & NS & NS & NS \\
\hline
\end{tabular}

Table 7: Crataegus oxyacantha spacing trial observation for seed weight (kg/plot).

\begin{tabular}{|c|c|c|c|c|c|}
\hline Spacing & $\begin{array}{l}\text { Seed weight } \\
\text { (kg)2007 }\end{array}$ & $\begin{array}{l}\text { Seed weight } \\
\text { (kg)2008 }\end{array}$ & $\begin{array}{l}\text { Seed weight } \\
\text { (kg)2009 }\end{array}$ & $\begin{array}{l}\text { Seed weight } \\
\text { (kg)2010 }\end{array}$ & $\begin{array}{c}\text { Seed weight } \\
(\mathrm{kg}) 2011\end{array}$ \\
\hline $\mathrm{S} 1=1 \times 1 \mathrm{~m}$ & 0.82 & 1.05 & 0.55 & 2.45 & 13.07 \\
\hline$S 2=2 \times 1 \mathrm{~m}$ & 1.03 & 1.62 & 0.39 & 5.88 & 11.82 \\
\hline $\mathrm{S} 3=2 \times 2 \mathrm{~m}$ & 0.79 & 1.30 & 0.93 & 7.73 & 13.02 \\
\hline $\mathrm{S} 4=3 \times 1 \mathrm{~m}$ & 0.81 & 1.23 & 0.89 & 4.23 & 11.30 \\
\hline $\mathrm{S} 5=3 \times 2 \mathrm{~m}$ & 0.97 & 1.53 & 0.34 & 7.12 & 11.33 \\
\hline SEm \pm & 0.49 & 0.36 & 0.10 & 1.27 & 2.01 \\
\hline $\mathrm{CD}$ at $5 \%$ & NS & NS & NS & NS & NS \\
\hline
\end{tabular}


Table 8: Crataegus oxyacantha spacing trial observation total seed weight (kg/plot).

\begin{tabular}{|c|c|c|c|c|c|}
\hline Spacing & $\begin{array}{l}\text { Seed weight } \\
\text { (kg) } 2012\end{array}$ & $\begin{array}{l}\text { Seed weight } \\
\text { (kg) } 2013\end{array}$ & $\begin{array}{l}\text { Seed weight } \\
(\mathrm{kg}) 2014\end{array}$ & $\begin{array}{l}\text { Seed weight } \\
(\mathrm{kg}) 2015\end{array}$ & $\begin{array}{l}\text { Seed weight } \\
\text { (kg)2016 }\end{array}$ \\
\hline $\mathrm{S} 1=1 \times 1 \mathrm{~m}$ & 19.52 & 19.17 & 5.83 & 47.00 & 5.33 \\
\hline $\mathrm{S} 2=2 \times 1 \mathrm{~m}$ & 14.13 & 20.17 & 7.17 & 50.67 & 6.33 \\
\hline$S 3=2 \times 2 m$ & 20.17 & 21.33 & 7.25 & 50.00 & 9.33 \\
\hline $\mathrm{S} 4=3 \times 1 \mathrm{~m}$ & 14.55 & 15.17 & 3.92 & 40.67 & 4.67 \\
\hline $\mathrm{S} 5=3 \times 2 \mathrm{~m}$ & 23.37 & 27.68 & 10.67 & 54.67 & 8.33 \\
\hline SEm \pm & 5.48 & 4.64 & 2.29 & 13.65 & 1.98 \\
\hline $\mathrm{CD}$ at $5 \%$ & NS & NS & NS & NS & NS \\
\hline
\end{tabular}

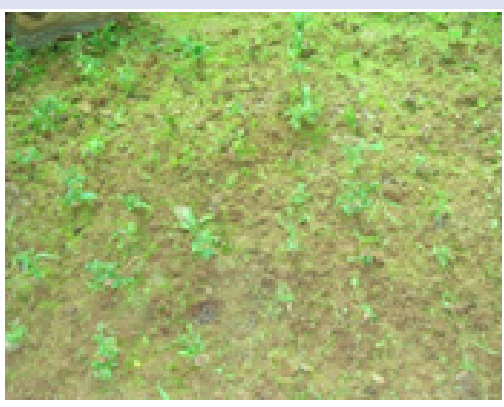

A. Nursery raisings by stem cuttings.

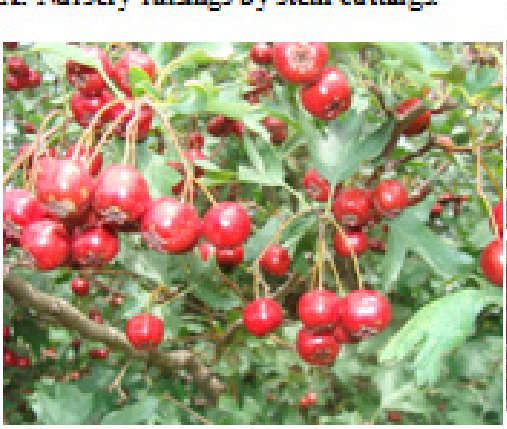

C. Mature fruiting in the field.

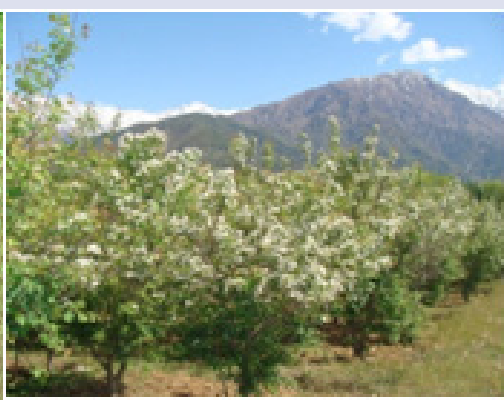

B. Flowering stage of Coutaegus in the field.

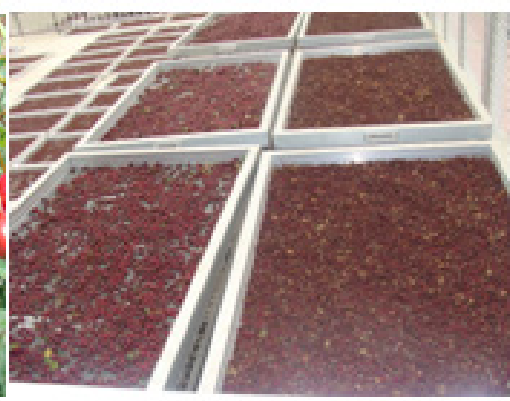

D. Seed drying in trays under shade.

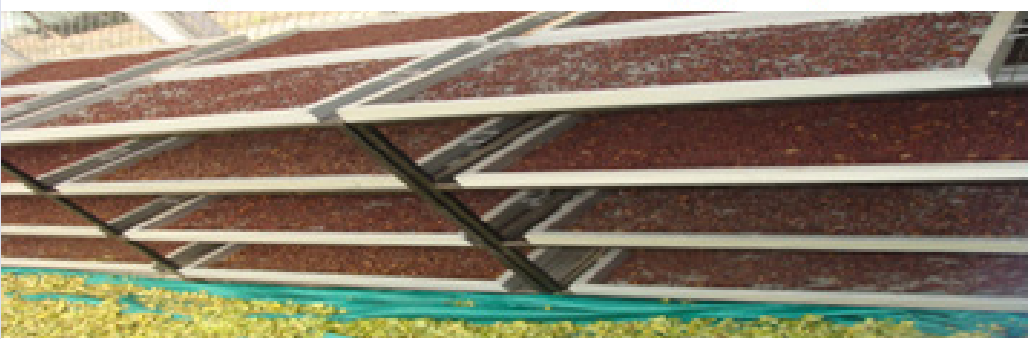

\section{E. L arge scale seed drying in aluminium trays under shade structure.}

Figure 1: Vegetative propagation by stem cuttings, R\&D field experiment and mature staging of fruit, different stage of seed drying under shade of $C$. oxyacantha.

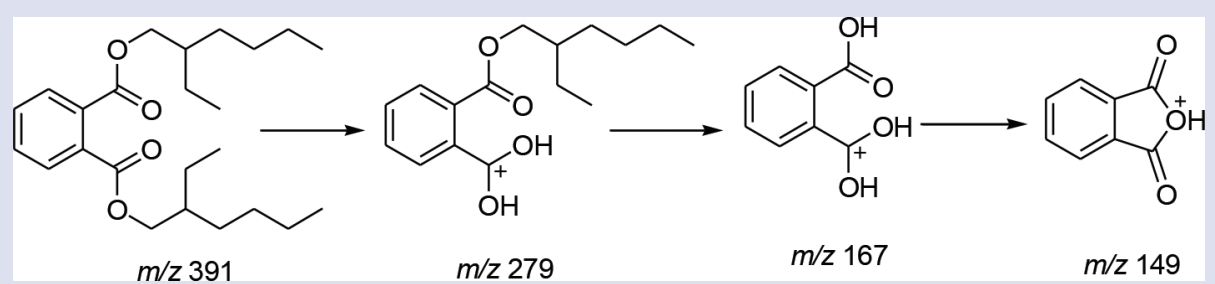

Figure 2: Mass fragmentation of 5 . 
Table 9: ${ }^{1} \mathrm{H}(300 \mathrm{MHz})$ and ${ }^{13} \mathrm{C}$ NMR $(75.4 \mathrm{MHz})$ spectral data of 5 in $\mathrm{CDCl}_{3}$.

\begin{tabular}{cccccc}
\hline Position & $\delta_{\mathrm{H}}(m, J \mathrm{~Hz})$ & $\Delta \mathrm{c}$ & Position & $\delta_{\mathrm{H}}(\mathrm{m}, \mathrm{J} \mathrm{Hz})$ & $\delta \mathrm{c}$ \\
\hline 1 & $7.56(d d, 3.0$ and 6.0$)$ & 131.2 & 7 & $1.33-1.38(\mathrm{~m})$ & 30.7 \\
2 & $7.74(d d, 3.0$ and 6.0$)$ & 129.1 & 8 & $1.33-1.38(\mathrm{~m})$ & 29.3 \\
3 & - & 132.8 & 9 & $1.33-1.38(m)$ & 23.3 \\
4 & - & 168.0 & 10 & $0.89-0.96(m)$ & 14.3 \\
5 & $4.18-4.28(m)$ & 68.5 & 11 & $1.40-1.45(m)$ & 24.1 \\
6 & $1.60-1.70(m)$ & 39.1 & 12 & $0.89-0.96(m)$ & 11.3 \\
\hline
\end{tabular}

Table 10: ${ }^{1} \mathrm{H}\left(300 \mathrm{MHz}\right.$ ) and ${ }^{13} \mathrm{C}$ NMR (75.4 MHz) spectral data of 9 in $\mathrm{CD}_{3} \mathrm{OD}$.

\begin{tabular}{cccccc}
\hline Position & $\delta_{\mathrm{H}}(m, J \mathrm{~Hz})$ & $\Delta \mathrm{c}$ & Position & $\delta_{\mathrm{H}}(m, J \mathrm{~Hz})$ & $\delta \mathrm{c}$ \\
\hline 2 & $5.10(d, 9.0)$ & 84.3 & $2^{\prime}$ & $6.72(\mathrm{~m})$ & 116.4 \\
3 & $4.72(d, 9.0)$ & 78.1 & $3^{\prime}$ & - & 148.0 \\
4 & - & 196.3 & $4^{\prime}$ & - & 147.1 \\
5 & - & 164.8 & $5^{\prime}$ & $6.91(\mathrm{~m})$ & 116.9 \\
6 & $5.90(d, 3.0)$ & 97.0 & $6^{\prime}$ & $6.72(\mathrm{~m})$ & 121.5 \\
7 & - & 169.6 & $1^{\prime \prime}$ & $3.82(d, 4.0)$ & 103.7 \\
8 & $5.90(d, 3.0)$ & 98.0 & $2^{\prime \prime}$ & $3.20(\mathrm{~m})$ & 76.4 \\
9 & - & 164.1 & $3^{\prime \prime}$ & $3.33(\mathrm{~m})$ & 74.0 \\
10 & - & 103.2 & $4^{\prime \prime}$ & $3.52(\mathrm{~m})$ & 71.5 \\
$1^{\prime}$ & - & 129.6 & $5^{\prime \prime}$ & $3.91(\mathrm{~m})$ & 66.0 \\
\hline
\end{tabular}

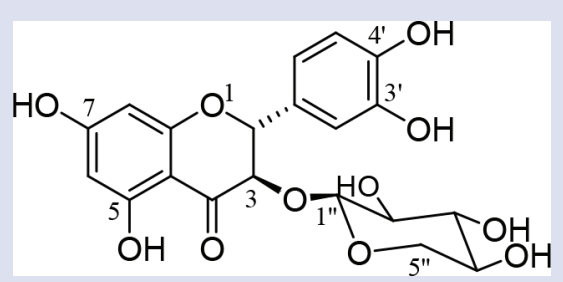

Figure 3: Structure of 9.

known isolated compounds are vitexin-2"-O-rhamnoside, isoquercitrin, $(2 R, 3 R)$ - taxifolin, oleanolic acid, ursolic acid, $\beta$-sitosterol, quercetin, rutin and $\beta$-sitosterol-3-O- $\beta$-D-glucoside.

\section{Bis-(2-ethylhexyl) phthalate (5)}

The compound $\mathbf{5}$ was obtained as reddish-brown precipitate from the ethyl acetate fraction of fruit of C. oxyacantha. The ESI-MS (+ve) spectrum showed a molecular ion peak at $\mathrm{m} / z 391[\mathrm{M}+\mathrm{H}]^{+}$corresponding to the molecular formula $\mathrm{C}_{24} \mathrm{H}_{38} \mathrm{O}_{4}$. The fragments at $\mathrm{m} / z 279,167$ and 149 with the consecutive loss of aliphatic chains were obtained according to the fragmentation pattern as shown in Figure 2.

The ${ }^{1} \mathrm{H}$ NMR spectrum showed two aromatic protons at $\delta_{\mathrm{H}} 7.74(d d, J=3.0$ and 6.0) and $7.56(d d, J=3.0$ and 6.0) integrating for two protons each. In addition, multiples in the aliphatic region were also observed. The ${ }^{13} \mathrm{C}$ NMR spectrum showed signals for 12 carbons including two methyl's, five methylene's, three methane's and two quaternary carbons. The presence of only two aromatic protons with ortho and meta coupling double doublets suggested that the compound must have an ortho-disubstituted benzene ring bearing the same substituent in both positions. The aromatic proton at $\delta_{\mathrm{H}} 7.74(d d, J=3.0$ and 6.0) showed HMQC corre- lation with the carbon at $\mathrm{C}-2\left(\delta_{\mathrm{C}} 129.1\right)$, while $\mathrm{HMBC}$ correlations with that of C-1 $\left(\delta_{\mathrm{C}} 131.2\right), \mathrm{C}-3\left(\delta_{\mathrm{C}} 132.8\right)$ and C-4 $\left(\delta_{\mathrm{C}} 168.0\right)$. The methylene multiple at $\delta_{\mathrm{H}} 4.18-4.28(\mathrm{H}-4, m)$ was attributed to C-5 $\left(\delta_{\mathrm{C}} 68.5\right)$ based on its $\mathrm{HMBC}$ correlations with neighbouring carbons. Its correlation with C-4 $\left(\delta_{\mathrm{C}} 168.0\right)$ indicated that it was linked to the carbonyl carbon through an oxygen atom i.e. has an ester linkage. The methyl multiple at $\delta_{\mathrm{H}}$ 0.89-0.96 (H-10 and $\left.12, m\right)$ integrating for twelve protons and correlations with methylene carbons at $\delta_{C} 23.3$ and $\delta_{C} 24.1$ were attributed to the positions C-10 $\left(\delta_{\mathrm{C}} 14.3\right)$ and C-12 $\left(\delta_{\mathrm{C}} 11.3\right)$. Hence, based on NMR (Table 9) and mass spectral data and its comparison with the literature values, ${ }^{9}$ the structure of $\mathbf{5}$ was established as bis-(2-ethylhexyl) phthalate. This is the first report on the isolation of this compound from the Crataegus spp.

\section{(2R,3R)-Taxifolin-3-O- $\beta$-D-xylopyranoside (9)}

The compound 9 was isolated as brown powder from fruit extract of C. oxyacantha. The ESI-MS spectrum displayed the molecular ion peak at $m / z 459[\mathrm{M}+\mathrm{Na}]^{+}$and $437[\mathrm{M}+\mathrm{H}]^{+}$corresponding to the molecular formula $\mathrm{C}_{20} \mathrm{H}_{20} \mathrm{O}_{11}$. One peak at $\mathrm{m} / z 306$ also appeared due to the loss of a pentose (132U). The NMR spectra of compound 9 were like that of taxifolin with the additional signals for a pentose Figure 3. The NMR values of the sugar were consistent with the xyloparyanoside.

The $\mathrm{H}-2$ and 3 protons resonated at $\delta_{\mathrm{H}} 5.10(d, J=9.0)$ and $4.72(d, J=9.0)$. According to data published in the literature (Hosoi et al., 2006), a coupling constant of 9.3-11.3 Hz correspond to trans form of taxifolin 3-O-arabinoside, which means that there are two possible configurations, either the $(+)$-trans form $(2 R, 3 R)$ or the $(-)$-trans form $(2 S, 3 S)$. The configuration was determined to be $2 R, 3 R$ by the optical rotation data i.e. $[\alpha]_{\mathrm{D}}{ }^{25}+20.9^{\circ}$, which was consistent with that of reported. ${ }^{10}$ The glycosylation at C-3 $\left(\delta_{\mathrm{C}} 78.1\right)$ was evident from the downfield shift of C-3 and the upfield shift of C-2 $\left(\delta_{C} 84.3\right)$ and C-4 $\left(\delta_{C} 196.3\right)$ due to $\beta$-effect. Hence, based on the spectral data (Table 10) and literature 
values, ${ }^{11}$ the structure of compound 9 was attributed to $(2 R, 3 R)$-taxifolin$3-O-\beta$-D-xylopyranoside. To the best of our knowledge this is the first report on the isolation of $(2 R, 3 R)$-taxifolin-3-O- $\beta$-D-xylopyranoside from Crataegus spp.

\section{DISCUSSION}

C. oxyacantha has a very high medicinal value and in this article, we have tried to standardize the propagation its techniques by using semi hard stem cuttings. Later in the field trial, the effect of different doses of FYM and various spacing on growth and biomass yield of seeds was studied. In the propagation trial, through stem cuttings, the statistically significant results were obtained in IBA when it was used in the concentration of $1000 \mathrm{mg} / \mathrm{l}$ and $1500 \mathrm{mg} / \mathrm{l}$ Table 2 . Due to pretreatment of stem cuttings by IBA, its endogenous level of hormones was increased due to availability of exposed surface area at the cut ends, which in turn promoted for the rooting. ${ }^{12}$ Similar finding has been reported, ${ }^{13,14}$ our results are fully in agreement to their findings. The hormones IAA, IBA, $\mathrm{GA}_{3}$ and Abscisic acid activate enzymes and mobilize reserve food material, which result in initiation of cell division, cell elongation and formation of callus in cut ends of stem cuttings, leading to its rooting. ${ }^{15,16}$ Our results are in agreement of findings, ${ }^{14}$ who observed stimulation of plant height, leaves numbers, root length by these hormone treatments in another Himalayan medicinal plant Taxus baccata. Some other workers have also reported the same type of findings. ${ }^{17,18}$

The plant growth was recorded from 2005 to 2009 continuously and then in 2015. It was observed that in early five years, it was growing and showing increasing trends. It was 65 to $80 \%$ higher in 2005 as compared to 2006 Table 3. While in 2007 it was $128 \%$ more than of 2008. From 2008 to 2009 , the growth was slightly slowed down to about $112 \%$. Again, it was reduced from 2009 to 2015 from 125 to $135 \%$ Table 3. It means after a certain period, the growth became stable in this plant as the energy and reserve food materials got utilized in different physiological, metabolic and seed production processes. The reserved food gets accumulate in the seeds and the overall growth status goes down.

In the different FYM applications, since 2008 onwards statistically significant results were obtained. The FYM, application has increased the overall production of seed Table 4,5. In middle, some year as 2014, 2016. The seed production was reduced drastically due to high wind, untimely rain and heavy hail storm at the time of flowering and seed formation. Especially, in the month of April and May, every year, these are natural calamities that highly affected seed yield. Most of the flowers fallen in soil due to high wind, rain and hail storm. The same type of studies carried out in our Institute. ${ }^{16,19}$ In most of the parameters various FYM application behave, separately plant species to species. In most of cases $22.5 \mathrm{t} /$ ha dose produced increasing trend in comparison to lower and higher doses of FYM Table 5,6. It was recorded that NPK are major nutrients, which play an important role in growth and yield of the crop. ${ }^{20,21}$ They further confirmed that a certain amount of nutrient (fertilizer) is required at a certain extent. Beyond a saturate stage, system did not allow it and no positive response was reported in terms of growth and yield. ${ }^{22}$ In our experiment after five years $2009,22.5 \mathrm{t} /$ ha produced higher plant growth, while in $1^{\text {st }}$ year lower doses are effective but higher dose $37.5 \mathrm{t} / \mathrm{ha}$ produced lower growth. Even in next five year means in 2015 lower dose $15 \mathrm{t} / \mathrm{ha}$ was produced better growth performance of plants. However, in terms of plant height all the results were statistically non-significant Table 6 . In terms of seed production again $22.5 \mathrm{t} /$ ha produced better yield in comparison to other doses in 2007 and 2008 years. After ten years in 2015 , in the end the statistically significant seed yield was recorded in $22.5 \mathrm{t} / \mathrm{h}$ a FYM application. In this year no any adverse of local weather or environmental conditions i.e. no high wind, no hail storm no heavy rains were recorded. But see in 2016 again the seed yield highly destructed by local environmental conditions caused low crop yield Table 7,8. It was reported that $50 \mathrm{~kg} \mathrm{~N}$ ha-1 was sufficient rather than higher doses. ${ }^{23,24}$ It has also recorded that excessive $\mathrm{N}$ supply reduced the yield and biomass. ${ }^{25}$

Therefore, our purpose was to develop agrotechniques for its domestication for the global society. This will reduce our dependency on these natural resources mean as farmers can grow it in their field following our recommendations.

\section{CONCLUSION}

The purpose of this study was to standardize the agrotechniques of cultivation and nursery raising of $C$. oxyacantha leading to yield and biomass production of the crop. Besides, the standardization of agrotechniques, its secondary metabolites have also been studied. Two new compounds have also been reported from its fruit extract. Chemical evaluation of seeds have also been compared based on its quality and quantity. For these study, some R \& D experiment were laid out. First of all, the nursery was raised from its semi hard stem cutting in conjunction with different concentrations of IAA, IBA, GA 3 and Abscisic acid hormones. Statistically significant sprouting was recorded in IBA- $1000 \mathrm{mg} / \mathrm{l}$ $78.35 \%$ followed by IBA $1000 \mathrm{mg} / \mathrm{l}$. For 10 years (2005 to 2015) duration, local climatic condition played an important role in seed yield as it got affected by high wind velocity, hail storms and high rainfall. Most of the flowers were fallen on ground surface. Spacing was not affected. Growth in plant height was not significant. Seed yield was statistically significant. FYM application showed its original activity and a saturations point. These parameters are statistically significant.

\section{ACKNOWLEDGEMENT}

Authors are grateful to National Medicinal Plant Board Govt. of India New Delhi and CSIR-IHBT for financial support and facilities. Authors are thankful to Er. Amit Kumar for providing help in manuscript preparation and editing.

\section{REFERENCES}

1. Long SR, Carey RA, Crofoot KM, Proteau PJ, Filtz TM. Effect of hawthorn (Crataegus oxycantha) crude extract and chromatographic fractions on multiple activities in a cultured cardiomyocyte assay. Phytomedicine. 2006;13(9):643-50

2. Jaya-Lakshmi R, Thirupurasundari CJ, Devaraj SN. Pretreatment with alcoholic extract of Crataegus oxycantha (AEC) activates mitochondrial protection during isoproterenol-induced myocardial infarction in rats. Molecular Cell Biochemistry. 2006;292(1):59-67.

3. Degenring FH, Suter A, Weber M, Saller R. A randomised double blind placebo controlled clinical trial of a standardised extract of fresh Crataegus berries (Crataegisan) in the treatment of patients with congestive heart failure NYHA II. Phytomedicine. 2003;10(5):363-9.

4. Sokol AL, Oszmianski WA. Antioxidant activity of phenolic compounds of hawthorn, pine, and skullcap. Food chem. 2007;103(3):853-9.

5. Tadic VM, Dobric S, Markovic G, Dordevic SM, Arsic IA, Menkovic NR et al. Anti-inflammatory, gastroprotective, free-radical-scavenging, and antimicrobial activities of hawthorn berries ethanol extract. J Agricultural Food Chem. 2008;56(17):7700-9.

6. Hanus M, Lafon J, Mathieu M. Double-blind, randomised, placebo-controlled study to evaluate the efficacy and safety of a fixed combination containing two plant extracts (Crataegus oxyacantha and Eschscholtzia californica) and magnesium in mild-to- moderate anxiety disorders. Current Med Res. Opinion. 2004;20(1):63-71.

7. Verma SK, Jain V, Verma D, Khamesra R. Crataegus oxyacantha- a cardioprotective herb. J Herb Med Toxicology. 2007;1(1):65-71.

8. Gulzaar-Sheikh. Medicinal Plants Introduction Centre Jammu and Kashmir. http//jkmpic.blogspot.com. 2017.

9. Al-Bari MAA, Sayeed MA, Rahman MS, Mossadik MA. Characterization and antimicrobial activities of a phthalic acid derivative produced by Streptomyces bangladeshiensis a novel species collected in Bangladesh. Research Journal of Medicine and Medical Sciences. 2006;1:77-81.

10. Claudia ASP. Investigation of anti-plasmodial compounds from various plant extracts. Ph.D. Thesis. University of Geneva, Geneva, Switzerland. 2009

11. Hosoi S, Shimizu E, Ohno K, Yokosawa R, Kuninaga S, Coskun M, et al. Struc- 
tural studies of zoospore attractants from Trachelospermum jasminoides var. pubescens: taxifolin 3-O-glycosides. Phytochemical Analysis. 2006;17(1):20-4.

12. Krishnamoorthy HN. Plant Growth substances including application in agriculture. Tata Mc Grew Hill Pub. Co. Ltd. New Delhi. 1981;214.

13. Kling GJ, Meyer MNJR. Effect of phenolic compounds and indole-acetic acid on adventitious root initiation in cuttings of Phaseolus aureus, Acer saccharinum and Acer griseum. Horticultural Science. 1983;81B:352-4.

14. Nandi SK, Rikhari HC, Nadeem M, Palni LMS. Clonal propagation of Taxus baccata L. a Himalayan asset under threat. Physiology Molecular Biology Plants. 1997;3:15-24.

15. Haissag BE. Influence of auxins and auxin synergists of adventitious primordial initiation and development. NZ J For Sci. 1986;4:311-27.

16. Gopichand, Meena RL. Standardization of propagation and agrotechniques in Ginkgo biloba L. A medicinally important plant. Journal of medicinal plants studies. 2015;3(4):6-15.

17. Van-Staden J, Hutton MJ, Drewes S. Cytokinin's in the leaves of G. Biloba L. The complex in mature leaves, Plant Physiol. 1983;73(2):223-7.

18. West WC, Frattarelli FJ, Russin KJ. Effect of stratification and gibberellins on seed germination in G. bilobaTorrey-Bot-Club-Bull. 1970;97(6):380-4.
19. Gopichand, Singh RD, Meena RL, Kaul VK, Singh B. Influence of manure and plant spacing on growth and yield of Dioscorea deltoidea Wall: an endangered species. Journal of Medicinal Plants Studies. 2013;1(3):184-90.

20. Carasky RJ, Lwuafor ENO. Contribution of soil fertility research and maintenance to improve maize production and productivity in Sub-Saharan African. Proceeding of regional Maize workshop on strategy for sustainable maize production in west and central Africa, April 21-25, International Institute for Tropical Agriculture (IITA)- Cotonous. Benin Republic. 1999;3-20.

21. Gopichand, Meena RL, Nag M, Pathania VL, Kaul VK, Singh B, et al. Effect of organic manure and plant spacing on biomass and quality of Mentha piperita $\mathrm{L}$. in Himalaya in India, Journal of essential oil research. 2013;25(4):354-7.

22. Buries RH. Nitrogen nutrition. Ann. Rev. Plant Physiol. 1959;20:301-8.

23. Krarup CA, Pertierra-Krurup R, Uragami A. Growth of Asperagus crowns with increasing nitrogen rates at three different sites. Proceeding of the 10th International Asparagus Symposium Niigata. Japan. 2002; pp:145-50.

24. Hussain AF, Anjum RA, Sajid M. Effect of nitrogen on the growth and yield of Asparagus (Asparagus officinalis). J Agric Biological Sci. 2006;1:41-7.

25. Paschold PJ, Hermann G, Artell B. Nitrogen, yields spear quality and N. Min Residues of Asparagus. 1999;35(10):588-92.

\section{ABOUT AUTHORS}

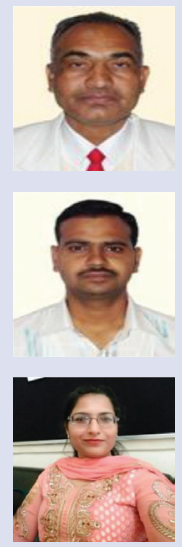

Gopichand: Sr. Principal Scientist in CSIR-IHBT, Palampur H.P. -176061, India

R.L. Meena: Technical Officer in CSIR-IHBT, Palampur H.P. - 176061, India

Pushpinder Kaur: Assistant Professor in Chemistry at Sri Guru Gobind Singh College, Sector-26, Chandigarh-160019, India.

\section{SUMMARY}

- Domestication of endangered and threatened species, Crataegus oxycantha.

- Fruiting at lower altitude, while the crop is of high altitude.

- Standardization of agrotechniques.

- Two new compounds was reported.

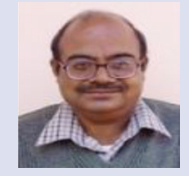

R.D. Singh: Late Chief Scientist in CSIR-IHBT, Palampur H.P. - 176061, India

Cite this article: Gopichand, Meena RL, Kaur P, Singh RD. Standardization of Agrotechniques and Biochemical Assessment of Crataegus oxyacantha in Western Himalaya. Pharmacog J. 2017;9(6):s69-s76. 